\title{
How To Manage Intracranial Arterial Stenosis? I'm Medical Or Interventional Treatment?
}

Kohansal Rahim, MD,

Neurologist,Neurointerventionist, Golestan University of M edical Sciences, Iran.

*Corresponding Author: Email: kohansal.ra@gmail.com

Background and review: The risk of stroke per year is about 3.9-28.7\% in patients with stenosis of the intracranial internal carotid artery and about $2.8-12 \%$ in patients with stenosis of middle cerebral artery. Intracranial stenoses are dynamic lesions. They can show progression, can remain stable or even undergo regression. The treatment of these patients is still controversial and several questions have to be answered.for example,whether only anti platelet/anticoagulantion therapy or angioplasty/stenting is the best first-line therapy?what is the best or maximal medical therapy?furthermore,what is the risk of stroke with medical or with interventional therapy or, what is the risk of complication from interventional therapy?is stenting necessary but or is angioplasty alone sufficient? Annual risk of stroke in patients with symptomatic intracranial stenosis where anti platelet/anticoagulantion therapy failed is about $4-12 \%(-45 \%)$. The intracranial complication rates of intracranial angioplasty or stenting are as high as $20 \%$.during treatment,ischemic stroke has been reported in $0-20 \%$,intracranial hemorrhage in $0-4 \%$,vessel dissection in $0-10 \%$,vessel rupture in $0-4 \%$, vessel occlusion/thrombosis in $0-4 \%$ and death in $0-8 \%$.

Conclusion: In patients with intracranial areterial stenosis angioplasty or stenting placement should not be the first-line therapy. Instead,initial treatment should include the standard control of vascular risk factors and application of antiplatelet/anticoagulantion drugs,statins and angiotensin convert ing enzym ihibitor.

Key words: Arterial Stenosis, Interventional Treatment

DOI: $10.7575 /$ aiac.abcmed.ca1.57

Published Date: February 2017

Peer-review is under responsibility of the 9th Iranian Stroke Congress.

Published by Australian International Academic Centre, Australia

This published work is open access under the CC BY license.

Available online at www.abcmed.aiac.org.au 